\title{
Left atrial appendage closure in a patient with atrial fibrillation affected by Rendu-Osler Weber disease
}

\begin{abstract}
Atrial fibrillation is the most common cardiac arrhythmia worldwide and represents a major risk factor for cerebral embolic stroke. The standard therapy in the prevention of stroke is oral anticoagulation therapy. However, a considerable number of patients are unable to support chronic OAC. Among these are patients with hereditary hemorrhagic telangiectasia. We present a case of a female patient affected by Rendu-OslerWeber disease who developed permanent atrial fibrillation with a CHA2DS2-VASc risk score of three. OAC was indicated despite her high bleeding risk. Therefore, an increase of muco-cutaneous and nose bleedings (BARC 2) with several admissions to the Emergency Department and multiple cauterizations influenced a worsening of her quality of life. On that basis OAC was discontinued.
\end{abstract}

After a multidisciplinary evaluation, we decided to perform percutaneous intervention of left atrial appendage closure with implantation of Watchman device. As anti-platelet post procedural therapy, considering her high bleeding risk, we adopted single anti-thrombotic strategy with clopidogrel $75 \mathrm{mg} /$ die, discontinued after three weeks due to increasing in recurrent nosebleeds, needed another cauterization. After 12 month follow up, the patient is in good health, with rare episodes of minor bleedings and a successful and well positioning of the intra-cardiac device, without thrombotic apposition, was evidenced at following echocardiographic examinations. In conclusion percutaneous intervention of LAA closure is confirmed to be an alternative and successful strategy in high bleeding risk patient with a contraindication to OAC. By thorough assessment, a single anti-thrombotic therapy with clopidogrel $75 \mathrm{mg} / \mathrm{die}$, after device implantation and for a time-limited period, might be considered.

Keywords: Atrial appendage - Rendu-Osler Weber disease - Atrial fibrillation

Abbrevations: OAC: Oral Anticoagulation; ROWD: Rendu-Osler Weber disease; HHT: Hereditary hemorrhagic telangiectasia; AF: Atrial fibrillation; ESC: European Society of Cardiology; DAPT: Dual antiplatelet therapy; ACP: Amplatzer cardiac plug; TGF $\beta$ : Transforming growth factor beta; VKA: Vitamin K antagonists;

\section{Introduction}

Atrial fibrillation represents the major risk factor for cardio-embolic events which the most frequent is cerebral stroke. Oral anticoagulation $(\mathrm{OAC})$ is considered the standard therapy in the prevention of cardio-embolic complications related to this arrhythmia. However, a substantial number of patients present contraindications to OAC because of high bleeding risk. Among these are patients affected by Rendu-Osler Weber disease (ROWD), also known as hereditary hemorrhagic telangiectasia (HHT). It is an autosomal dominant inherited disease with complete penetrance in adulthood, characterized by multifocal widening of distal arteries and a high prevalence of vascular malformations due to a defect in the TGF $\beta$ pathway that led an aberrant angiogenesis.

The diagnosis of ROW disease is made around 40-50 years old and it is based on the presence of at least three of four Curacao criteria [1].

They are: Spontaneous and recurrent epistaxis, Family history, Cutaneous and mucosal teleangectasie

Arterovenous malformations of parenchymatous organs.

In HHT hemorragical events could lead to
Davide Bosi*, Fabiana Cozza, Daniela Lina, Angela Guidorossi, Alberto Menozzi

U.O. di Cardiologia, Azienda Ospedaliera Universitaria di Parma, Italy *Author for correspondence: Email: bosidavide88@gmail.com Received date: August 11, 2018 Accepted date: October 11, 2018 Published date: October 17, 2018 
a severe anemia with a progressive decrease in quality of life.

The most common clinical manifestation is nosebleed with a prevalence ranging from $78 \%$ to $96 \%$ [2], gastrointestinal telangiectasia are present in the $80 \%$ of this population [3] and $23 \%$ of the patients have cerebral arterovenous malformations with an annual risk of rupture and bleeding of $0.5 \%$ [4].

As a result, patients with ROW disease have an increased risk of life threatening bleedings. Thus, these groups of patients have a relative or absolute contraindication for antithrombotic therapy.

\section{Case Report}

We present a case of a 75 years old female, affected by Rendu-Osler-Weber disease diagnosed at the age of 47 on the basis of recurrent episodes of epistaxis, oral mucosa bleeding and diffuse cutaneous telangiectasia (Figure 1) and first degree relatives with the same disease. Arteriovenous malformations were not detected on total body CT-scan. A mild hypertension and hypercholesterolemia, not in pharmacological treatment, were her only cardiovascular risk factors.

From 2014 she had permanent atrial fibrillation (AF), without symptoms and hemodynamically stable. Transthoracic echocardiography exam revealed a dilated left atrium with moderate mitral regurgitation due to posterior leaflet prolapse. Accordingly, no attempts of electrical or pharmacological cardioversion were taken into consideration. However, her CHA2DS2-VASc risk score was three, giving her an estimated annual cardioembolic risk of 4.6\% [5]. On that basis, long term systemic oral anticoagulation (OAC) was warranted despite of the haemorrhagic risk due to Rendu- Osler Weber disease. The internist clinician chose warfarin therapy, with close monitoring of INR target range to 2-2.5, while NOACs were not considered, given the impossibility of INR control and dose modulation. However, she had an increase of muco-cutaneous and nose bleedings (BARC 2) with several admissions to the Emergency Department and multiple cauterizations that influenced a worsening of her quality of life.

After one year, OAC therapy was discontinued. Nevertheless, cardio embolic stroke risk was persistently high and, after multidisciplinary evaluation (cardiologist and internist), we considered an alternative, nonpharmacological, method for reducing the risk of further cerebrovascular events. Thus, we proposed to our patient a percutaneous intervention of left atrial appendage (LAA) closure in order to avoid long term anticoagulation. Finally, in May 2017 patient was operated for LAA closure intervention using fluoroscopy and transesophageal echocardiography guidance (Figures 2 and 3), with the implantation of Watchman LAA occlusion device N 21. The procedure was well tolerated without any complications. As post-procedural antithrombotic therapy, considering her high bleeding risk, we decided to administer single antiplatelet therapy with clopidogrel $75 \mathrm{mg} /$ die for only one month.

Three weeks after the discharge, the patient was admitted to ED for severe nose bleeding (BARC 2) treated with another cauterization. Then the antiplatelet therapy was discontinued in advance.

Twelve months follow-up she is in good health, with occasional episodes of minor bleedings. The successful and well positioning of the intra-cardiac device was evidenced at following echocardiographic examinations. In conclusion, our patient is satisfied and she declared an improvement in her quality of life, similar to that before atrial fibrillation diagnosis.

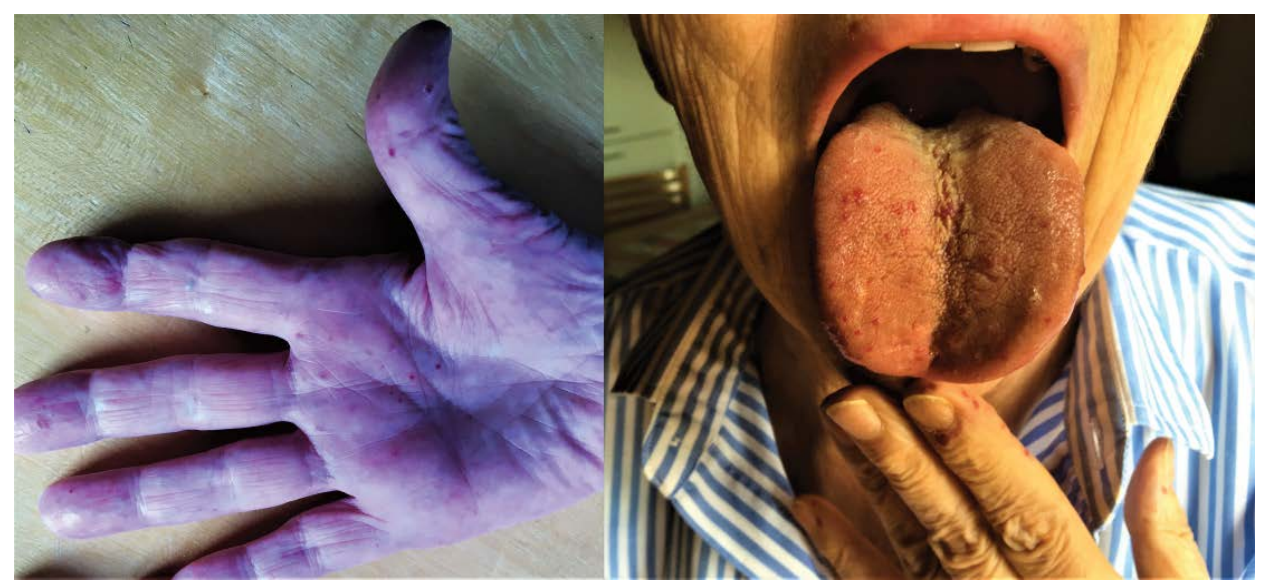

Figure 1. Cutaneous and tongue teleangectasie of our patient. 

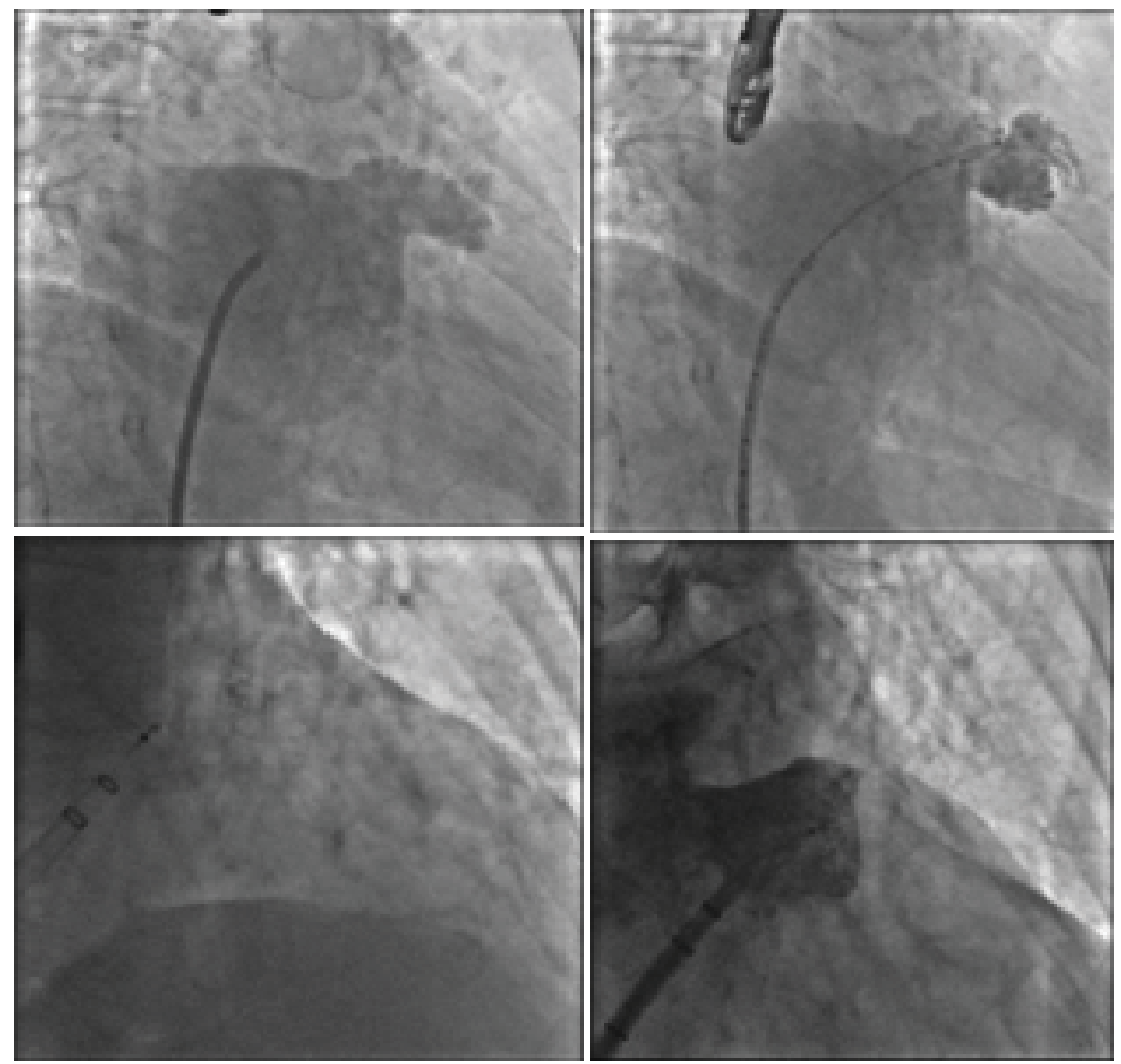

Figure 2. Fluroscopy images of some moments of the procedure.
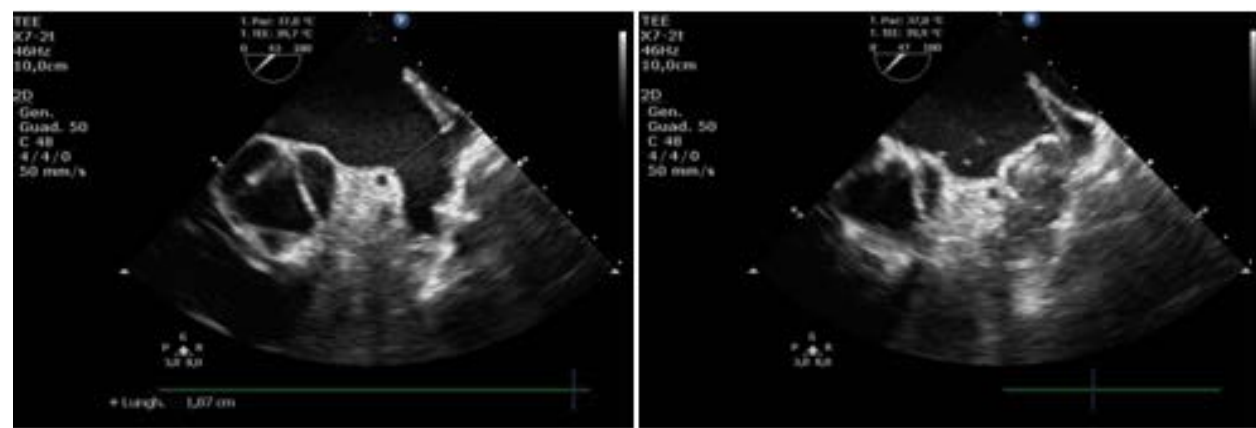

Figure 3. Transesophageal ultrasound before and after positioning of the device.

\section{Discussion}

The management of patients with high risk of bleeding, such as those affected by Rendu-Osler-Weber disease, and concomitant thromboembolic risk due to the presence of atrial fibrillation represents a challenging clinical scenario.

Currently, in this category of patients there are no guidelines that indicate the best therapeutic strategy. Edwards et al. showed that most patients can tolerate anticoagulation, with only a $20 \%$ rate of cessation because severe bleeding complication [6]. Based on online survey, Devlin et al. demonstrated that in more of $50 \%$ of ROWD patients were advised by a doctor not to use anticoagulation or antiplatelet agents; therefore, this study indicates unexplained and wide variation in the side effect profiles with respect to hemorrhage associated with the use of anticoagulant and antiplatelet therapies in patients with HHT [7].

Even in the case of our patient, after an initial period of therapy with VKA, this treatment was suspended 
because of a sharp increase in muco-cutaneous bleedings, although this choice exposed the patient to a non-negligible thromboembolic risk.

Percutaneous closure of the left appendage was proven to be a valid therapeutic strategy, alternative to OAC. The PROTECT-AF trial demonstrated the safety and efficacy of this procedure, through use of the WATCHMAN Device, in preventing thromboembolic events compared with warfarin therapy [8,9]. Afterwards, the PREVAIL study showed the same good results pointing out a reduction in peri-procedural complications compared to PROTECT-AF trial $(4.2 \%$ vs. $8.7 \%)[10]$.

Another device used in clinical practice is the AMPLATZER Cardiac Plug (ACP), and its evolution Amulet [11], which has shown in several studies excellent results in terms of both effectiveness and safety [12-14].

There are still several controversies regarding the choice of antithrombotic therapy and its duration after device implantation. In the PROTECT-AF trial, the anticoagulation protocol adopted was OAC for 6 weeks, DAPT for 6 months, and ASA for life. All patients enrolled in this study had to be eligible for warfarin to enable randomization to either a WATCHMAN device occlusion or chronic warfarin therapy. It is obvious that this therapeutic strategy in patients with contraindications to anticoagulant therapy, as is the case of our patient, can be dangerous. Subsequently, the ASAP study demonstrated that DAPT alone for 6 months after the left atrial appendage closure with the WATCHMAN device was efficacious and safe [15], suggesting that anticoagulant therapy may not be necessary.

The above-mentioned studies concerning the use of the Amplatzer Cardiac Plug device were performed by administering an initial period of DAPT, ranging from 1 to 6 months, then continuing with a single

\section{References}

1. CL Shovlin, AE Guttmacher, E Buscarini, et al. Diagnostic criteria for hereditary hemorrhagic telangiectasia (Rendu OslerWeber syndrome). Am J Med Genet. 91(1): 66-67 (2000).

2. H Plauchu, JP Chadarevian, A Bideau, et al. Age-related clinical profile of heredity hemorrhagic telangiectasia in an epidemiologically recruited population. Am J Med Genet. 32(3): 291-297 (1989).

3. AV Longacre, CP Gross, M Gallitelli, et al. Diagnosis and antiplatelet drug.

On that basis, in 2014 the European Society of Cardiology published a Consensus Statement where the DAPT, from one to six month, is suggested, followed by lifelong single antiplatelet therapy [16]. However, more recent studies propose a reduction in the overall duration of dual antiplatelet therapy or try to explore the possibility of starting directly with a monotherapy with aspirin or clopidogrel in relation to the high risk of bleeding of patients selected for the procedure [1719]. Our clinical case is in agreement with the result showed in these last studies, outlining the possibility of reducing the duration of antiplatelet therapy, necessary to promote proper endothelialization of the device, without loss of efficacy and exposing these patients to a lower risk of bleeding.

\section{Conclusion}

Our case report is a further demonstration of the efficacy and safety of the left atrial appendage clousure, as an alternative strategy in atrial fibrillation patients with a controindication to OAC due to high bleeding risk. Indeed, ESC guide lines suggest to taking into consideration the percutaneous intervention in this group of patients, even though with a low level of evidence at the moment (Class of recommendation IIb, level of evidence B) [20].

In our opinion, in this group of patients is essential a multidiscliplinary evaluation, in order to developed a tailored management. In contrast with the 2014 European Consensus Statement, in our patient we decided to administer a very short single antiplatelet therapy, without any device-related thromboembolic complications at one year follow-up. In data, there are not reported trials supporting a very short antiplatelet monotherapy, but only single clinical experiences [21]. Further studies are needed to establish the optimum antithrombotic therapy after percutaneous left atrial appendage closure in high bleeding risk patients.

management of gastrointestinal bleeding in patients with hereditary hemorrhagic telangiectasia. Am J Gastroenterol. 98(1): 59-65 (2003).

4. RB Willemse, JJ Mager, CJJ Westermann, et al. Bleeding risk of cerebrovascular malformations in hereditary hemorrhagic telangiectasia. J Neurosurg. 92(5): 779-784 (2000).

5. Friberg L, Rosenqvist M, Lip GY. Evaluation of risk stratification schemes for ischemic stroke and bleeding in 182678 patients with atrial fibrillation: the Swedish Atrial Fibrillation cohort study. Eur Heart J. 33(12): 1500-1510 (2012). 
6. Edwards CP, Shehata N, Faughnan ME. Hereditary hemorrhagic telangiectasia patients can tolerate anticoagulation. Ann Hematol. 91(12): 1959-1968 (2012).

7. Devlin HL, Hosman AE, Shovlin CL. Antiplatelet and Anticoagulant Agents in Hereditary Hemorrhagic Telangiectasia. N Engl J Med. 368: 876-878 (2013).

8. Fountain RB, Holmes DR, Chandrasekaran K, et al.The PROTECT AF (WATCHMAN Left Atrial Appendage System for Embolic PROTECTion in Patients with Atrial Fibrillation) trial. Am Heart J. 151(5): 956-961 (2006).

9. Holmes DR, Reddy VY, Turi ZG, et al. PROTECT AF Investigators. Percutaneous closure of the left atrial appendage versus warfarin therapy for prevention of stroke in patients with atrial fibrillation: a randomised non-inferiority trial. Lancet. 374(9689): 534-542 (2009).

10. Holmes DR, Kar S, Price MJ, et al. Prospective Randomized Evaluation of the Watchman Left Atrial Appendage Closure Device in Patients With Atrial Fibrillation Versus Long-Term Warfarin Therapy: the PREVAIL trial. J Am Coll Cardiol. 64(1): 1-12 (2014).

11. Freixa X, Chan JL, Tzikas A, et al. The Amplatzer Cardiac Plug 2 for left atrial appendage occlusion: novel features and first-inman experience. Euro Intervention. 8: 1094-1098 (2014).

12. Urena M, Rodes-Cabau J, Freixa X, et al. Percutaneous left atrial appendage closure with the AMPLATZER cardiac plug device in patients with non valvular atrial fibrillation and contraindications to anticoagulation therapy. J Am Coll Cardiol. 62: 96-102 (2013)

13. Park JW, Bethencourt A, Sievert $\mathrm{H}$, et al. Left atrial appendage closure with Amplatzer Cardiac Plug in atrial fibrillation: initial European experience. Catheter Cardiovasc Interv. 77(5): 700-716 (2011).
14. Nietlispach F, Gloekler S, Krause R, et al. Amplatzer left atrial appendage occlusion: single center 10-year experience. Catheter Cardiovasc Interv. 82: 283-9 (2013).

15. Reddy VY, Mobius-Winkler S, Miller MA, et al. Left atrial appendage closure with the Watchman device in patients with a contraindication for oral anticoagulation: the ASAP study (ASA Plavix Feasibility Study With WatchmanLeft Atrial Appendage Closure Technology). J Am Coll Cardiol. 61: 2551-2556 (2013).

16. Meier B, Blaauw Y, Khattab A, et al. EHRA/EAPCI expert consensus statementon catheter-based left atrial appendage occlusion. Europace. 16: 1397-1416 (2014).

17. Jalala Z, Dineta M L, Combesb N, et al. Percutaneous left atrial appendage closure followed by single antiplatelet therapy: Shortand mid-term outcomes. Arch Cardiovasc Dis. 16: 1875-1883 (2017).

18. Rodriguez-Gabella T, Nombela-Franco L, Regueiro A, et al. Single Antiplatelet Therapy Following Left Atrial Appendage Closure in Patients with Contraindication to Anticoagulation. J Am Coll Cardiol. 68: 1920-1921 (2016).

19. Tzikas A, Shakir S, Gafoor S, et al. Left atrial appendage occlusion for stroke prevention in atrial fibrillation: multicentre experience with the AMPLATZERCardiac Plug. Euro Intervention. 11: 1170-1179 (2015).

20. Kirchhof P, Benussi S, Kotecha D, et al. 2016 ESC Guidelines for the management of atrial fibrillation developed in collaboration with EACTS. Eur Heart J. 37: 2893-962 (2016).

21. Spina R1, Gunalingam B. Left atrial appendage occlusion with the Watchman device in a patient with paroxysmal atrial fibrillation and intolerance of all forms of anticoagulation due to hereditary haemorrhagic telangiectasia. Intern Med J. 44(3):2957 (2014). 